\title{
Association of Sugar-Sweetened Beverage Frequency with Adiposity: Evidence from the "Children of 1997" Birth Cohort
}

\author{
Ting Zhang ${ }^{1}$, Shiu Lun Au Yeung ${ }^{1} \mathbb{D}$, Man Ki Kwok ${ }^{1} \mathbb{C}$, Lai Ling Hui ${ }^{1,2}$, \\ Gabriel Matthew Leung ${ }^{1}$ and C. Mary Schooling ${ }^{1,3, *}$ \\ 1 School of Public Health, Li Ka Shing Faculty of Medicine, The University of Hong Kong, Hong Kong SAR, \\ China; zhangt77@connect.hku.hk (T.Z.); ryanaysl@connect.hku.hk (S.L.A.Y.); maggiek@hku.hk (M.K.K.); \\ huic@hku.hk (L.L.H.); gmleung@hku.hk (G.M.L.) \\ 2 Department of Pediatrics, Faculty of Medicine, The Chinese University of Hong Kong, \\ Hong Kong SAR, China \\ 3 CUNY School of Public Health and Health Policy, New York, NY 10027, USA \\ * Correspondence: cms1@hku.hk; Tel.: (852) 3917 6732; Fax: (852) 35201945
}

Received: 12 March 2020; Accepted: 3 April 2020; Published: 7 April 2020

\begin{abstract}
Background: Observationally, sugar-sweetened beverage (SSB) consumption is associated with adiposity in Western children but could be confounded. We examined the association of SSB frequency with adiposity in the non-Western setting of Hong Kong. Methods: We examined the associations of SSB consumption frequency at 11 and 13 years assessed by using a food frequency questionnaire with subsequent body mass index (BMI) z-score and overweight/obesity up to 18 years using generalized estimating equations, and with waist circumference, waist-to-hip ratio, and body fat percentage at 16-19 years using linear regression in a population-representative Chinese birth cohort "Children of 1997" ( $n=3628)$. Results: At 11 and 13 years, 6.8\% and 8.2\% of children respectively consumed SSB daily. Neither SSB frequency at 11 nor at 13 years was associated with subsequent BMI z-score or overweight/obesity up to 18 years, or with waist circumference, waist-to-hip ratio, or body fat percentage at 16-19 years adjusted for age, sex, socioeconomic position, health status, physical activity and other food consumption, although bias to the null from under-reporting cannot be eliminated. Conclusion: Although we cannot definitively exclude a small association of SSB frequency with adiposity, lack of association of SSB frequency with adiposity in a non-Western setting with low SSB consumption suggests that the role of SSB in adiposity appears to be minor.
\end{abstract}

Keywords: adiposity; children; sugar-sweetened beverages

\section{Background}

Mean body mass index (BMI) and the prevalence of obesity in children and adolescents increased dramatically from 1975 to 2016 [1]. Added sugars, particularly in the form of sugar-sweetened beverages (SSB), contribute to excess calories, and may play a role in the development of obesity, because sugars in liquid form may induce less satiety than in solid form [2] and promote the over-consumption of calories. Health organizations, including the World Health Organization (WHO) and the American Heart Association, have recommended restricting SSB intake to address the obesity epidemic $[3,4]$. Meta-analyses of observational studies mainly from Western settings have suggested higher risk of weight gain or becoming overweight or obese among children with higher intake of SSBs [5,6]. However, observational studies are susceptible to unmeasured confounding by socioeconomic position (SEP), unhealthy lifestyle, and health status, making the interpretation uncertain [7]. Meta-analyses of observational studies not corrected for multiple testing or multiple modeling may be biased and 
unreliable [8]. A few randomized controlled trials (RCT) have been conducted in children $[9,10]$, which suggested reducing SSB intake reduces weight. However, meta-analyses of RCTs have revealed mixed findings. One meta-analysis showed no overall effect of SSB intake on weight in children, possibly due to low compliance in some included trials [6]. This meta-analysis also found weight decrease for reduced sugars intake and weight increase for increased sugars intake in trials of adults with ad libitum diets [6]. However, some others showed small effects of SSB intake on weight or BMI, which may be more marked for increasing consumption of SSB on weight gain and among the overweight $[5,11,12]$. As a beverage containing naturally occurring sugars, $100 \%$ fruit juice was not associated with weight gain in children aged 7 to 18 years in a meta-analysis of observational studies [13], but no RCT to date has tested the effect of $100 \%$ fruit juice on adiposity in children.

Given the inconclusive evidence concerning the effect of SSB on adiposity, assessing the association in settings with different confounding patterns can be helpful [14]. Unlike in Western developed settings, obesity is less clearly socially patterned in Hong Kong Chinese children [15,16]. Chinese children also consume fewer SSBs than those in Western developed countries [17]. SSB consumption in Chinese children may have different socioeconomic patterns than in Western settings, where children of lower SEP tend to consume more SSB [18]. Here, we examined the association of SSB frequency at 11 and 13 years with subsequent BMI z-score and overweight (including obesity) up to 18 years and waist circumference (WC), waist-to-hip ratio (WHR) and body fat percentage (BFP) at 16 to 19 years in the non-Western developed setting of Hong Kong using a large, population-representative Hong Kong Chinese birth cohort "Children of 1997".

\section{Methods}

\subsection{The "Children of 1997" Birth Cohort}

The "Children of 1997" birth cohort is a population-representative Chinese birth cohort ( $n=8327)$ that included 88.0\% of all births in Hong Kong from April 1, 1997 to May 31, 1997, as described in detail elsewhere [19]. Newborns were recruited at their first postnatal visit to any of the 49 Maternal and Child Health Centers (MCHC) in Hong Kong. Baseline characteristics, including maternal and infant characteristics (birth weight, birth order, maternal age at birth, delivery mode), socioeconomic characteristics (mother's birthplace, highest parental education), and early life factors (breastfeeding), were assessed using a self-administered questionnaire in Chinese at recruitment. Highest parental occupation, type of housing tenure and household income were also recorded. Passive follow-up via record linkage was instituted in 2005 to obtain all routine annual measurements of height and weight from age 6 years onwards from the Student Health Service (SHS). Height without shoes was measured by stadiometer to the nearest $0.1 \mathrm{~cm}$, and weight without shoes and outer clothing was measured by calibrated digital scales to the nearest $0.1 \mathrm{~kg}$. A postal survey (Survey I) was sent in July 2008, then re-sent a second and third time as necessary to non-respondents over the following 9 months. The survey in Chinese (or English if requested) included questions on food frequency (e.g., fruit, vegetable, meat, SSB), physical activity, general health, main caregiver, and WC (with measuring tape and detailed instruction) of the child. One feature of our setting is that the calculated calorie intakes seemed to be within plausible ranges [20]. A postal survey follow-up (Survey II) was conducted in 2010-2012, which included similar questions on food frequency. The Biobank Clinical Follow-up in 2013-2016 included measurements of height, weight, WC, hip circumference and BFP according to standard protocols. Weight and BFP were measured using bioelectrical impedance analysis (BIA) with a Tanita segmental body composition monitor (Tanita BC-545, Tanita Co., Tokyo, Japan). WC and hip circumference were measured by trained technicians using a standard protocol. Ethical approval for the study, including comprehensive health-related analyses, was obtained from the Institutional Review Board of the University of Hong Kong/Hospital Authority Hong Kong West Cluster (HKU/HA HKW IRB). 


\subsection{Exposure-SSB Consumption}

SSB consumption at 11 years was assessed by asking parents in Survey I "On average, how often did your child drink canned pop/soda/soft drinks in the last week?" with responses "never ever drinks" $(n=162,4.5 \%)$, "not at all in the last week" $(n=1039,28.6 \%), " 1-3$ times a week" $(n=1841,50.7 \%)$, "4-6 times a week" ( $n=340,9.4 \%)$, "once a day" $(n=193,5.3 \%)$, "2-3 times a day" $(n=40,1.1 \%)$, and " 4 or more times a day" $(n=13,0.4 \%)$. Reponses were combined into the following categories: "less than weekly", "1-3 times per week", "4-6 times per week" and "daily" because of the relatively small numbers in some categories. The food frequency questionnaire in Survey I showed some validity regarding the consumption of milk and non-milk dairy products [21]. We also assessed SSB consumption at 13 years by asking children the same question with the same responses in Survey II. We did not assess the consumption of specific types of SSB, therefore the reported intake of SSB included beverages sweetened with sugar and beverages sweetened with non-nutritive sweeteners.

\subsection{Outcomes-BMI, WC, WHR and BFP}

The primary outcomes were age and sex-specific BMI z-scores relative to the 2007 WHO growth reference for 5-19 years [22] at each age from 12 to 18 years. Overweight and obesity were defined using the 2000 International Obesity Task-force (IOTF) cut-offs [23], i.e., overweight is equivalent to an adult BMI of $\geq 25$ to $<30 \mathrm{~kg} / \mathrm{m}^{2}$, and obesity to an adult BMI of $\geq 30 \mathrm{~kg} / \mathrm{m}^{2}$. We also calculated BMI $z$-scores and centiles using the 2012 IOTF reference [24]. Due to the relatively small number regarding obesity $(\sim 4 \%)$, overweight and obese were combined. The secondary outcomes were WC $(\mathrm{cm}), \mathrm{WHR}$, and BFP (\%) at 16-19 years (mean age at measurement $17.5 \pm 0.5$ years).

\subsection{Statistical Analysis}

In the "Children of 1997" birth cohort, we used chi-squared tests and Cohen effect sizes (Cohen's $\mathrm{w}$ effect size for categorical variables, 0.1 for small, 0.3 for medium, and 0.5 for large) [25] to compare participants with and without information on SSB at 11 years. Cohen effect sizes in contrast to chi-squared tests are independent of sample size. We also used one-way analysis of variance (ANOVA) or chi-squared tests to assess the relation of potential confounders with SSBs intake. We used multivariable generalized estimating equations (GEE) with an "exchangeable" (i.e., assuming correlation between measurements were consistent over time) working correlation structure to assess the adjusted associations of SSB consumption at 11 years with BMI z-score and overweight (including obesity) from 12 to 18 years, and we used multivariable linear regression models to assess the associations of SSB consumption at 11 years with WC, WHR and BFP at 16-19 years. Confounders, considered as common causes of SSB consumption and adiposity, were age, sex, maternal age at birth, mother's birthplace, highest parental education, highest parental occupation, household income per head, main caregiver, general health status, physical activity, and fruit, vegetable and meat consumption and BMI z-score at 11 years. We present four models. Model 1 adjusted for age and sex. Model 2 additionally adjusted for maternal age at birth, mother's birthplace, highest parental education, highest parental occupation, household income per head and the interaction of mother's birthplace and highest parental education [15]. Model 3 additionally adjusted for main caregiver, general health status, and physical activity. Model 4 additionally adjusted for fruit, vegetable, and meat consumption and BMI z-score at 11 years.

To handle missing data, we combined multiple imputation (MI) and inverse probability weighting (IPW). We used multiple imputation for missing confounders (among 3300 with exposure and at least one measurement of BMI from 12 to 18 years, maternal age at birth for less than $0.1 \%$, mother's birthplace for $0.3 \%$, highest parental occupation for $12.8 \%$, household income per head for $11.2 \%$, main caregiver for $0.7 \%$, general health status for $3.2 \%$, physical activity for $11.2 \%$, fruit consumption for $0.3 \%$, vegetable consumption for $0.5 \%$, meat consumption for $0.5 \%$, and BMI z-score at 11 years for $18.4 \%$ ) based on a flexible additive regression model with predictive mean matching incorporating 
data on sex, birth order, maternal age at birth, mother's birthplace, father's birthplace, highest parental education, highest parental occupation, household income per head, main caregiver, general health status, physical activity, fruit, vegetable, and meat consumption and BMI z-score at 11 years, SSB consumption, and outcomes (BMI z-scores from 12 to 18 years, WC, WHR or BFP). We imputed missing data 20 times using the "Hmisc" R package. We calculated the probability of observations being present using logistic regression models to identify potential predictors of missingness, from which inverse probability weights of response were obtained for each observation. We analyzed the 20 imputed datasets using these weights and summarized the results into single estimated beta-coefficients or odds ratios (OR) with 95\% confidence intervals (CI) adjusted for missing data uncertainty [26]. We repeated the analyses by combining $\mathrm{MI}$ with inverse probability weighting (IPW) [27]. For comparison, we also conducted a complete case analysis. Given the potential under-reporting of SSB frequency, as a sensitivity analysis we repeated the complete case analyses after randomly reallocating some of those reporting "less than weekly", "1-3 times per week" and "4-6 times per week" to a higher frequency category to evaluate the effect on the association of SSB consumption with overweight (including obesity). We also assessed the associations of SSB consumption at 13 years with BMI z-score and overweight (including obesity) from 14 to 18 years by using GEE, and with WC, WHR and BFP at 16-19 years by using multivariable linear regression models adjusting for age, sex, maternal age at birth, maternal birthplace, parental highest education level, parental highest occupation, household income per head, and interaction of maternal birthplace with parental highest education level in a complete case analysis.

All statistical analyses were performed using R version 3.6.1 (The R Foundation for Statistical Computing, Vienna, Austria).

\section{Results}

Of the 8327 individuals originally recruited to the "Children of 1997" birth cohort, as of December 2018, 28 had permanently withdrawn. Of the remaining 8299, 7934 were potentially contactable for Survey I in 2008-2009, whilst 75 migrated without trace, 12 deceased, and 278 were untraceable (probably migrated or dead). Of these 7934, 3678 responded to Survey I, of whom 98.6\% provided information on SSB consumption at about 11 years (mean age at assessment $11.5 \pm 0.3$ years) (Figure S1). The proportion of English responses was very low $(7 / 3678=0.2 \%)$. The 3628 children with information on SSB consumption and 4670 children without were significantly different relating to some characteristics, but the magnitude of the difference was small for sex, maternal age at birth, mother's place of birth, highest parental education, highest parental occupation, and household income per head (all Cohen effect sizes <0.1) [25] (Table S1). At about 11 years, 33.1\% had not consumed SSB during the past week, 50.7\% consumed 1-3 times during the past week, 9.4\% consumed 4-6 times, and $6.8 \%$ consumed daily. The mean of BMI at 11 years was $18.55 \pm 3.24 \mathrm{~kg} / \mathrm{m}^{2}$ and BMI z-score was $0.21 \pm 1.27$. Table 1 shows that boys, those with younger mothers, mothers born in Hong Kong, or those had $\geq 1 \mathrm{~h} /$ day of physical activity, less frequent fruit and vegetable consumption, and more frequent meat consumption were more likely to consume SSB. SSB consumption was not related to SEP (parental education, parental occupation, and household income). 
Table 1. Characteristics by sugar-sweetened beverage (SSB) consumption at 11 years for 3628 children in Hong Kong's "Children of 1997" birth cohort.

\begin{tabular}{|c|c|c|c|c|c|c|c|}
\hline \multirow{2}{*}{ Characteristics } & \multirow{2}{*}{ Items } & \multirow{2}{*}{$n$} & \multicolumn{4}{|c|}{ SSB Frequency at 11y (row \%) } & \multirow{2}{*}{$p$-Value * } \\
\hline & & & <weekly & 1-3 times/week & 4-6 times/week & Daily & \\
\hline \multirow[t]{2}{*}{ Sex } & Girls & 1863 & 37.5 & 48.2 & 8.4 & 5.9 & $<0.001$ \\
\hline & Boys & 1765 & 28.4 & 53.4 & 10.4 & 7.8 & \\
\hline \multirow[t]{4}{*}{ Maternal age at birth } & $<=24 y$ & 346 & 27.2 & 51.7 & 11.0 & 10.1 & 0.04 \\
\hline & $25-29 y$ & 1129 & 32.6 & 51.4 & 9.6 & 6.5 & \\
\hline & $30-34 y$ & 1438 & 35.2 & 49.5 & 9.5 & 5.8 & \\
\hline & $>=35 y$ & 713 & 32.4 & 51.9 & 8.0 & 7.7 & \\
\hline \multirow[t]{2}{*}{ Mother's birthplace } & Non-HK & 1419 & 38.7 & 47.1 & 7.8 & 6.4 & $<0.001$ \\
\hline & HK & 2200 & 29.5 & 53.2 & 10.4 & 7.0 & \\
\hline \multirow[t]{3}{*}{ Highest parental education } & Grade 9 or below & 1029 & 32.4 & 50.9 & 9.2 & 7.5 & 0.64 \\
\hline & Grade $10-11$ & 1558 & 32.9 & 50.3 & 9.7 & 7.1 & \\
\hline & Grade 12 or above & 1041 & 34.2 & 51.2 & 9.0 & 5.6 & \\
\hline \multirow[t]{6}{*}{ Highest parental occupation } & Professional & 842 & 34.0 & 51.1 & 9.6 & 5.3 & 0.10 \\
\hline & Managerial & 469 & 34.3 & 48.4 & 10.7 & 6.6 & \\
\hline & $\begin{array}{l}\text { Non-manual } \\
\text { skilled }\end{array}$ & 918 & 29.6 & 54.1 & 9.4 & 6.9 & \\
\hline & Manual skilled & 518 & 35.7 & 46.3 & 10.8 & 7.1 & \\
\hline & Semiskilled & 318 & 29.9 & 52.2 & 8.8 & 9.1 & \\
\hline & Unskilled & 93 & 41.9 & 48.4 & 6.5 & 3.2 & \\
\hline \multirow[t]{5}{*}{ Household income ${ }^{\#}$} & 1st quintile & 588 & 35.5 & 47.6 & 8.8 & 8.0 & 0.13 \\
\hline & 2nd quintile & 626 & 32.9 & 51.6 & 9.9 & 5.6 & \\
\hline & 3rd quintile & 630 & 32.7 & 48.7 & 11.3 & 7.3 & \\
\hline & 4th quintile & 670 & 29.9 & 54.9 & 8.1 & 7.2 & \\
\hline & 5th quintile & 699 & 34.8 & 50.4 & 9.7 & 5.2 & \\
\hline \multirow[t]{3}{*}{ Main care giver } & Parents & 2985 & 33.8 & 49.8 & 9.3 & 7.1 & 0.15 \\
\hline & Grandparents & 224 & 27.7 & 57.6 & 10.3 & 4.5 & \\
\hline & Other & 393 & 31.6 & 53.2 & 9.9 & 5.3 & \\
\hline \multirow[t]{3}{*}{ General health } & Good & 2927 & 32.5 & 51.3 & 9.1 & 7.1 & 0.21 \\
\hline & Average & 515 & 36.9 & 48.0 & 10.3 & 4.9 & \\
\hline & Poor & 68 & 38.2 & 45.6 & 8.8 & 7.4 & \\
\hline \multirow[t]{2}{*}{ Physical activity at $11 \mathrm{y}$} & $<1 \mathrm{~h} / \mathrm{d}$ & 2282 & 34.5 & 49.8 & 8.9 & 6.7 & 0.02 \\
\hline & $\geq 1 \mathrm{~h} / \mathrm{d}$ & 943 & 29.3 & 53.7 & 10.6 & 6.5 & \\
\hline \multirow[t]{4}{*}{ Fruit consumption at $11 \mathrm{y}$} & $<$ weekly & 131 & 35.1 & 41.2 & 14.5 & 9.2 & $<0.001$ \\
\hline & $1-3$ times & 970 & 27.2 & 53.2 & 11.4 & 8.1 & \\
\hline & 4-6 times & 667 & 29.7 & 54.0 & 9.6 & 6.7 & \\
\hline & Daily & 1847 & 37.0 & 49.2 & 7.9 & 5.8 & \\
\hline
\end{tabular}


Table 1. Cont.

\begin{tabular}{|c|c|c|c|c|c|c|c|}
\hline \multirow{2}{*}{ Characteristics } & \multirow{2}{*}{ Items } & \multirow{2}{*}{$n$} & \multicolumn{4}{|c|}{ SSB Frequency at 11 y (row \%) } & \multirow{2}{*}{$p$-Value } \\
\hline & & & $<$ weekly & 1-3 times/week & 4-6 times/week & Daily & \\
\hline \multirow[t]{4}{*}{$\begin{array}{l}\text { Vegetable consumption at } \\
11 \mathrm{y}\end{array}$} & $<$ weekly & 69 & 20.3 & 49.3 & 14.5 & 15.9 & $<0.001$ \\
\hline & $1-3$ times & 325 & 25.8 & 51.4 & 12.3 & 10.5 & \\
\hline & $4-6$ times & 414 & 28.0 & 51.7 & 11.1 & 9.2 & \\
\hline & Daily & 2802 & 35.0 & 50.7 & 8.6 & 5.7 & \\
\hline \multirow[t]{4}{*}{ Meat consumption at $11 \mathrm{y}$} & $<$ weekly & 27 & 51.9 & 40.7 & 3.7 & 3.7 & $<0.001$ \\
\hline & $1-3$ times & 459 & 40.3 & 46.4 & 5.9 & 7.4 & \\
\hline & $4-6$ times & 662 & 31.1 & 57.1 & 7.6 & 4.2 & \\
\hline & Daily & 2459 & 32.1 & 49.9 & 10.7 & 7.3 & \\
\hline \multirow[t]{4}{*}{ SSB frequency at $13 y$} & <weekly & 902 & 48.7 & 44.7 & 4.3 & 2.2 & $<0.001$ \\
\hline & 1-3 times/week & 1279 & 29.3 & 56.6 & 9.5 & 4.6 & \\
\hline & 4-6 times/week & 310 & 18.7 & 54.2 & 16.1 & 11.0 & \\
\hline & Daily & 222 & 16.2 & 43.7 & 17.1 & 23.0 & \\
\hline BMI $\left(\mathrm{kg} / \mathrm{m}^{2}\right)$ at $11 \mathrm{y}$ & Mean (SD) & 2858 & $18.3(3.1)$ & $18.7(3.3)$ & $18.7(3.4)$ & $18.7(3.2)$ & 0.04 \\
\hline BMI z-score at $11 \mathrm{y}$ & Mean (SD) & 2858 & $0.12(1.20)$ & $0.25(1.29)$ & $0.23(1.34)$ & $0.28(1.25)$ & 0.04 \\
\hline \multirow[t]{3}{*}{ Weight status at $11 \mathrm{y}$} & Normal & 2266 & 35.0 & 49.9 & 9.2 & 5.9 & 0.06 \\
\hline & Overweight & 515 & 30.1 & 54.4 & 9.3 & 6.2 & \\
\hline & Obese & 77 & 23.4 & 53.2 & 16.9 & 6.5 & \\
\hline WC (cm) at 11y & Mean (SD) & 3184 & $66.4(8.7)$ & $66.8(9.1)$ & $67.3(9.6)$ & $66.9(9.4)$ & 0.13 \\
\hline BMI $\left(\mathrm{kg} / \mathrm{m}^{2}\right)$ at $12 \mathrm{y}$ & Mean (SD) & 2121 & $19.0(3.0)$ & $19.2(3.4)$ & $19.2(3.6)$ & $19.2(3.2)$ & 0.30 \\
\hline BMI z-score at $12 \mathrm{y}$ & Mean (SD) & 2121 & $0.13(1.13)$ & $0.18(1.27)$ & $0.15(1.32)$ & $0.24(1.14)$ & 0.36 \\
\hline BMI $\left(\mathrm{kg} / \mathrm{m}^{2}\right)$ at $13 \mathrm{y}$ & Mean (SD) & 2378 & $19.6(3.0)$ & $19.8(3.5)$ & $19.9(3.5)$ & $19.9(3.3)$ & 0.19 \\
\hline BMI z-score at $13 y$ & Mean (SD) & 2378 & $0.07(1.07)$ & $0.09(1.22)$ & $0.14(1.21)$ & $0.15(1.16)$ & 0.29 \\
\hline BMI $\left(\mathrm{kg} / \mathrm{m}^{2}\right)$ at $14 \mathrm{y}$ & Mean (SD) & 1925 & $19.9(2.9)$ & $20.1(3.4)$ & $20.3(3.4)$ & $19.9(2.9)$ & 0.29 \\
\hline BMI $z$-score at $14 \mathrm{y}$ & Mean (SD) & 1925 & $-0.09(1.02)$ & $-0.07(1.15)$ & $0.02(1.21)$ & $-0.07(1.07)$ & 0.42 \\
\hline BMI $\left(\mathrm{kg} / \mathrm{m}^{2}\right)$ at $15 \mathrm{y}$ & Mean (SD) & 1658 & $20.4(3.1)$ & $20.4(3.4)$ & $20.5(3.0)$ & $20.3(3.4)$ & 0.89 \\
\hline BMI z-score at $15 y$ & Mean (SD) & 1658 & $-0.15(1.04)$ & $-0.17(1.15)$ & $-0.10(1.07)$ & $-0.21(1.14)$ & 0.91 \\
\hline BMI $\left(\mathrm{kg} / \mathrm{m}^{2}\right)$ at $16 \mathrm{y}$ & Mean (SD) & 647 & $20.3(2.8)$ & $20.7(3.4)$ & $20.9(2.9)$ & $20.5(3.4)$ & 0.30 \\
\hline BMI z-score at $16 y$ & Mean (SD) & 647 & $-0.29(1.02)$ & $-0.22(1.12)$ & $-0.07(0.94)$ & $-0.27(1.13)$ & 0.38 \\
\hline BMI $\left(\mathrm{kg} / \mathrm{m}^{2}\right)$ at $17 \mathrm{y}$ & Mean (SD) & 1258 & $20.6(3.3)$ & $21.2(3.8)$ & $21.0(3.1)$ & $20.6(3.1)$ & 0.35 \\
\hline BMI $z$-score at $17 y$ & Mean (SD) & 1258 & $-0.38(1.08)$ & $-0.23(1.21)$ & $-0.25(1.08)$ & $-0.38(1.09)$ & 0.41 \\
\hline BMI $\left(\mathrm{kg} / \mathrm{m}^{2}\right)$ at $18 \mathrm{y}$ & Mean (SD) & 464 & $20.8(3.5)$ & $20.6(3.4)$ & $20.0(3.2)$ & $20.5(2.9)$ & 0.27 \\
\hline BMI z-score at $18 \mathrm{y}$ & Mean (SD) & 464 & $-0.45(1.15)$ & $-0.53(1.19)$ & $-0.71(1.15)$ & $-0.53(1.06)$ & 0.30 \\
\hline $\mathrm{WC}(\mathrm{cm})$ at $16-19 \mathrm{y}$ & Mean (SD) & 2134 & $71.3(9.0)$ & $72.4(9.3)$ & $71.9(9.1)$ & $71.8(7.7)$ & 0.23 \\
\hline WHR at $16-19 y$ & Mean (SD) & 2128 & $0.76(0.06)$ & $0.77(0.06)$ & $0.77(0.06)$ & $0.76(0.05)$ & 0.37 \\
\hline BFP $(\%)$ at $16-19 y$ & Mean (SD) & 2136 & $22.3(8.3)$ & $21.9(9.2)$ & $21.4(8.5)$ & $21.4(9.0)$ & 0.10 \\
\hline
\end{tabular}

BFP, body fat percentage; BMI: body mass index; SD: standard deviation; SSB, sugar-sweetened beverage; WC, waist circumference; WHR, waist-to-hip ratio. \# Mean (SD) for household income per head in quintiles were 1st quintile: HKD 1746 (SD 419), 2nd quintile: HKD 2853 (325), 3rd quintile: HKD 4365 (557), 4th quintile: HKD 6826 (883) and 5th quintile: HKD 14,945 $(15,620)(7.8 \mathrm{HKD}=1 \mathrm{USD}) .{ }^{*}$ Differences of continuous or categorical variables between SSB frequencies were evaluated by using one-way ANOVA or chi-squared tests, respectively. 
Table 2 shows that SSB consumption was neither associated with BMI z-score nor overweight (including obesity) from 12 to 18 years in all four models using MI/IPW. Repeating the analysis using a complete case analysis or using BMI z-score or centile based on the 2012 IOTF reference [24] in a complete case analysis produced similar results (Table S2). There were no clear dose-response associations of SSB consumption with WC, WHR or BFP at 16-19 years in all four models using MI/IPW (Table 3) or complete case analysis (Table S3). After considering potential under-reporting of SSB frequency, we found that a positive association of 1-3 times per week of SSB consumption with overweight was possible if $20 \%$ or a larger proportion of 1-3 times per week were underreported as less than weekly, or $15 \%$ or more of 1-3 times per week, 4-6 times per week and daily were underreported as a lower frequency category (Table S4). We also found no association of SSB consumption frequency at 13 years with measures of adiposity in a complete case analysis (Table 4). Using a composite measure of SSB intake at 11 and 13 years gave a similar interpretation (Table S5).

Table 2. Associations of sugar-sweetened beverage (SSB) consumption at 11 years with body mass index (BMI) z-score and overweight from 12 to 18 years in Hong Kong's "Children of 1997" birth cohort.

\begin{tabular}{|c|c|c|c|c|c|c|c|c|}
\hline \multirow{2}{*}{ Model } & \multirow{2}{*}{$\begin{array}{c}\text { SSB } \\
\text { Consumption }\end{array}$} & \multirow{2}{*}{$n^{\#}$} & \multicolumn{3}{|c|}{ BMI z-Score } & \multicolumn{3}{|c|}{ Overweight (Including Obesity) * } \\
\hline & & & Beta & SE & $95 \% \mathrm{CI}$ & OR & SE of logOR & $95 \%$ CI \\
\hline \multirow[t]{4}{*}{1} & <weekly & 1098 & Ref & & & Ref & & \\
\hline & 1-3 times/week & 1682 & 0.027 & 0.043 & $-0.058,0.111$ & 1.21 & 0.10 & $0.99,1.46$ \\
\hline & 4-6 times/week & 310 & 0.041 & 0.074 & $-0.105,0.187$ & 1.16 & 0.16 & $0.84,1.59$ \\
\hline & Daily & 210 & 0.042 & 0.081 & $-0.118,0.201$ & 1.05 & 0.19 & $0.72,1.53$ \\
\hline \multirow[t]{4}{*}{2} & $<$ weekly & 1098 & Ref & & & Ref & & \\
\hline & 1-3 times/week & 1682 & 0.027 & 0.043 & $-0.058,0.112$ & 1.21 & 0.10 & $0.99,1.46$ \\
\hline & 4-6 times/week & 310 & 0.042 & 0.074 & $-0.104,0.188$ & 1.17 & 0.16 & $0.85,1.61$ \\
\hline & Daily & 210 & 0.016 & 0.082 & $-0.144,0.176$ & 0.97 & 0.20 & $0.66,1.43$ \\
\hline \multirow[t]{4}{*}{3} & $<$ weekly & 1098 & Ref & & & Ref & & \\
\hline & 1-3 times/week & 1682 & 0.016 & 0.043 & $-0.069,0.101$ & 1.21 & 0.10 & $0.99,1.47$ \\
\hline & 4-6 times/week & 310 & 0.037 & 0.074 & $-0.109,0.182$ & 1.18 & 0.17 & $0.86,1.64$ \\
\hline & Daily & 210 & 0.000 & 0.081 & $-0.159,0.159$ & 0.97 & 0.20 & $0.66,1.43$ \\
\hline \multirow[t]{4}{*}{4} & $<$ weekly & 1098 & Ref & & & Ref & & \\
\hline & 1-3 times/week & 1682 & 0.004 & 0.021 & $-0.037,0.046$ & 1.16 & 0.14 & $0.88,1.52$ \\
\hline & 4-6 times/week & 310 & 0.037 & 0.035 & $-0.031,0.105$ & 1.05 & 0.22 & $0.68,1.63$ \\
\hline & Daily & 210 & -0.050 & 0.045 & $-0.138,0.037$ & 0.81 & 0.30 & $0.45,1.45$ \\
\hline
\end{tabular}

BMI: body mass index; SE: standard error; CI: confidence interval; OR: odds ratio; SSB, sugar-sweetened beverage. All values were derived from GEE models. Model 1 adjusted for age and sex; Model 2 additionally adjusted for maternal age at birth, maternal birthplace, parental highest education level, parental highest occupation, household income per head, and interaction of maternal birthplace with parental highest education level; Model 3 additionally adjusted for main caregiver, general health, and physical activity; Model 4 additionally adjusted for fruit, vegetable, and meat consumption and BMI z-score at 11 years. " Number of observations with SSB consumption, covariates, and at least one measurement of BMI. * Reference: normal weight. 
Table 3. Associations of sugar-sweetened beverage (SSB) consumption at 11 years with waist circumference (WC), waist-to-hip ratio (WHR) and body fat percentage (BFP) at 16-19 years in Hong Kong's “Children of 1997" birth cohort.

\begin{tabular}{|c|c|c|c|c|c|c|c|c|c|c|c|c|c|}
\hline \multirow{2}{*}{ Model } & \multirow{2}{*}{$\begin{array}{c}\text { SSB } \\
\text { Consumption }\end{array}$} & \multicolumn{4}{|c|}{ WC } & \multicolumn{4}{|c|}{ WHR } & \multicolumn{4}{|c|}{ BFP } \\
\hline & & $n$ & Beta & $\mathrm{SE}$ & $95 \% \mathrm{CI}$ & $n$ & Beta & $\mathrm{SE}$ & $95 \% \mathrm{CI}$ & $n$ & Beta & SE & $95 \% \mathrm{CI}$ \\
\hline \multirow[t]{4}{*}{1} & <weekly & 578 & Ref & & & 576 & Ref & & & 578 & Ref & & \\
\hline & 1-3 times/week & 846 & 0.85 & 0.48 & $-0.09,1.78$ & 843 & 0.003 & 0.003 & $-0.003,0.009$ & 845 & 0.60 & 0.34 & $-0.06,1.26$ \\
\hline & 4-6 times/week & 157 & -0.47 & 0.79 & $-2.01,1.07$ & 157 & -0.003 & 0.005 & $-0.012,0.007$ & 158 & -0.47 & 0.56 & $-1.56,0.62$ \\
\hline & Daily & 90 & 0.44 & 1.01 & $-1.55,2.42$ & 90 & -0.001 & 0.006 & $-0.013,0.011$ & 91 & 0.50 & 0.71 & $-0.90,1.90$ \\
\hline \multirow[t]{4}{*}{2} & $<$ weekly & 578 & Ref & & & 576 & Ref & & & 578 & Ref & & \\
\hline & 1-3 times/week & 846 & 0.97 & 0.48 & $0.03,1.91$ & 843 & 0.003 & 0.003 & $-0.002,0.009$ & 845 & 0.60 & 0.34 & $-0.07,1.27$ \\
\hline & 4-6 times/week & 157 & -0.44 & 0.79 & $-1.99,1.10$ & 157 & -0.003 & 0.005 & $-0.012,0.007$ & 158 & -0.54 & 0.56 & $-1.63,0.56$ \\
\hline & Daily & 90 & 0.37 & 1.02 & $-1.63,2.37$ & 90 & -0.002 & 0.006 & $-0.014,0.011$ & 91 & 0.33 & 0.72 & $-1.08,1.74$ \\
\hline \multirow[t]{4}{*}{3} & $<$ weekly & 578 & Ref & & & 576 & Ref & & & 578 & Ref & & \\
\hline & 1-3 times/week & 846 & 1.02 & 0.48 & $0.08,1.96$ & 843 & 0.004 & 0.003 & $-0.002,0.010$ & 845 & 0.63 & 0.34 & $-0.03,1.30$ \\
\hline & 4-6 times/week & 157 & -0.39 & 0.79 & $-1.93,1.15$ & 157 & -0.002 & 0.005 & $-0.012,0.007$ & 158 & -0.50 & 0.56 & $-1.59,0.59$ \\
\hline & Daily & 90 & 0.45 & 1.02 & $-1.54,2.44$ & 90 & -0.001 & 0.006 & $-0.014,0.011$ & 91 & 0.39 & 0.71 & $-1.01,1.79$ \\
\hline \multirow[t]{4}{*}{4} & $<$ weekly & 578 & Ref & & & 576 & Ref & & & 578 & Ref & & \\
\hline & 1-3 times/week & 846 & 0.60 & 0.38 & $-0.15,1.35$ & 843 & 0.002 & 0.003 & $-0.004,0.007$ & 845 & 0.40 & 0.26 & $-0.12,0.91$ \\
\hline & 4-6 times/week & 157 & -0.46 & 0.62 & $-1.68,0.76$ & 157 & -0.003 & 0.005 & $-0.012,0.006$ & 158 & -0.55 & 0.41 & $-1.36,0.26$ \\
\hline & Daily & 90 & -0.58 & 0.84 & $-2.23,1.08$ & 90 & -0.004 & 0.006 & $-0.016,0.008$ & 91 & -0.37 & 0.54 & $-1.43,0.69$ \\
\hline
\end{tabular}

BFP, body fat percentage; SE: standard error; CI: confidence interval; OR: odds ratio; SSB, sugar-sweetened beverage; WC, waist circumference; WHR, waist-to-hip ratio. All values were derived from multivariable linear regression models. Model 1 adjusted for age and sex; Model 2 additionally adjusted for maternal age at birth maternal birthplace, parental highest education level, parental highest occupation, household income per head, and interaction of maternal birthplace with parental highest education level; Model 3 additionally adjusted for main caregiver, general health, and physical activity; Model 4 additionally adjusted for fruit, vegetable, and meat consumption and BMI z-score at 11 years. 
Table 4. Associations of sugar-sweetened beverage (SSB) consumption at 13 years with body mass index (BMI) z-score and overweight from 14-18 years and with waist circumference (WC), waist-to-hip ratio (WHR) and body fat percentage (BFP) at 16-19 years in Hong Kong's "Children of 1997" birth cohort.

\begin{tabular}{|c|c|c|c|c|c|}
\hline Outcomes & SSB Consumption & $n$ & Beta/OR * & $\mathrm{SE}^{+}$ & $95 \%$ CI \\
\hline \multirow[t]{4}{*}{ BMI z-score } & <weekly & 924 & Ref & & \\
\hline & 1-3 times/week & 1230 & -0.04 & 0.05 & $-0.13,0.06$ \\
\hline & 4-6 times/week & 283 & 0.04 & 0.07 & $-0.11,0.18$ \\
\hline & Daily & 180 & 0.00 & 0.09 & $-0.18,0.18$ \\
\hline Overweight & <weekly & 924 & Ref & & \\
\hline \multirow[t]{3}{*}{ (including obesity) \# } & 1-3 times/week & 1230 & -0.08 & 0.12 & $-0.32,0.16$ \\
\hline & 4-6 times/week & 283 & -0.15 & 0.20 & $-0.53,0.24$ \\
\hline & Daily & 180 & -0.19 & 0.23 & $-0.64,0.27$ \\
\hline \multirow[t]{4}{*}{ WC } & $<$ weekly & 521 & Ref & & \\
\hline & 1-3 times/week & 669 & 0.11 & 0.51 & $-0.89,1.11$ \\
\hline & 4-6 times/week & 155 & 0.17 & 0.80 & $-1.40,1.74$ \\
\hline & Daily & 108 & 0.89 & 0.93 & $-0.93,2.71$ \\
\hline \multirow[t]{4}{*}{ WHR } & $<$ weekly & 520 & Ref & & \\
\hline & $1-3$ times & 667 & 0.000 & 0.003 & $\begin{array}{c}-0.006 \\
0.006\end{array}$ \\
\hline & $4-6$ times & 153 & 0.001 & 0.005 & $\begin{array}{c}-0.009 \\
0.010\end{array}$ \\
\hline & Daily & 108 & 0.005 & 0.006 & $\begin{array}{c}-0.006 \\
0.016\end{array}$ \\
\hline \multirow[t]{4}{*}{ BFP } & $<$ weekly & 521 & Ref & & \\
\hline & $1-3$ times & 668 & 0.20 & 0.36 & $-0.50,0.91$ \\
\hline & $4-6$ times & 155 & 0.41 & 0.56 & $-0.68,1.51$ \\
\hline & Daily & 108 & 0.90 & 0.65 & $-0.37,2.17$ \\
\hline
\end{tabular}

BFP, body fat percentage; BMI: body mass index; SE: standard error; CI: confidence interval; OR: odds ratio; SSB, sugar-sweetened beverage; WC, waist circumference; WHR, waist-to-hip ratio. Values for BMI z-score and overweight were derived from GEE models, whereas values for WC, WHR and BFP were derived from multivariable linear regression models. All models were adjusted for age, sex, maternal age at birth, maternal birthplace, parental highest education level, parental highest occupation, household income per head, and interaction of maternal birthplace with parental highest education level. \# Reference: normal weight. * Beta for BMI z-score, WC, WHR and BFP and OR for overweight (including obesity). ${ }^{\dagger}$ Standard error of logOR for overweight (including obesity).

\section{Discussion}

In this large, population-representative birth cohort of Hong Kong Chinese children, SSB frequency at 11 or 13 years was not associated with subsequent BMI z-score or overweight (including obesity) up to 18 years, nor with WC, WHR or BFP at 16-19 years.

To our knowledge, this is the first study to examine the association of SSB consumption with adiposity using a prospective cohort of Chinese children. Cross-sectional studies in Chinese children $[17,28]$ have shown a positive association of regular SSB consumption with obesity and abdominal obesity in 6-13-year-olds [28] and an association of SSB consumption with abdominal but not general obesity in 6-17-year-olds [17]. Our lack of association of SSB consumption frequency with subsequent BMI z-score or other measures of adiposity differs from an observational study [29] and meta-analyses of observational studies largely from Western settings [5,6,30,31]. A cohort study conducted in US children suggested that BMI z-score was 0.050 higher (95\% CI 0.022 to 0.079 ) for each additional $8 \mathrm{oz} /$ day of SSB consumed at ages 2 to 17 years [29]. The discrepancy between our study and that of Marshall et al. [29] may be because the participants in our study had a much lower SSB intake (6.8\% consumed SSB daily at 11 years) than those in that study (median 8 oz/day at 11 years). Notably, Marshall et al. also found a positive association of water/sugar-free beverages with BMI z scores [29], thus confounding may exist. In the US, both SSB intake and adiposity are strongly associated with socio-economic position, which could confound the estimates [15,16]. The social patterning of SSB consumption and of obesity is less marked in our Hong Kong Chinese setting [15], making our observations less open to confounding. Our finding of no association of SSB frequency with subsequent BMI z-score is inconsistent with two RCTs in children, showing that reducing SSB 
intake reduces weight $[9,10]$. A possible explanation is that subjects in the two trials had high daily SSB intake at baseline, thus the findings may not apply to individuals with a low SSB intake. Our finding of little association of SSB consumption frequency with adiposity is more consistent with the limited evidence from meta-analyses of RCTs of SSB showing minor effects on obesity $[5,6,11,12]$ which are more pronounced in overweight individuals.

SSB is the largest source of added sugars in US children [32], although the contribution of SSB to added sugars intake has declined and is no longer the largest source in some countries, such as the Germany [33]. SSB may contribute to obesity because sugar in liquid form adds to calories but induces less satiety than in solid form and may weaken any compensatory reduction in subsequent calorie intake and facilitate over-consumption of calories [2]. However, a recent experimental study in mice found no difference in adiposity by sucrose intake but did find differences by fat intake [34], suggesting that fat rather than sucrose drives over-consumption. Observationally, consumption of $100 \%$ fruit juice, which may contain as much sucrose as SSB, is unrelated to weight gain in children aged 7-18 years despite slight weight gain in younger children, although this observation has not been confirmed in an RCT [13]. Instead, the observed association of SSB with adiposity in Western populations could be due to modern patterns of sugary/fatty snacking characterized by high SSB consumption, rather to sugar itself [35].

Despite being conducted in a setting where diet has been reported quite accurately in terms of total calories [36], and confounding may be less marked [15], our study has several limitations. First, SSB consumption was obtained by a food frequency questionnaire and may be underreported due to social desirability bias [37]. Non-differential misclassification of SSB frequency usually biases towards the null, which could have obscured a positive association of SSB with overweight if $\geq 20 \%$ of those drinking SSB 1-3 times/week underreported as less than weekly, or $\geq 15 \%$ of those drinking SSB 1-3 times/week, 4-6 times/week and daily were underreported as a lower category. Baseline SSB consumption may subsequently change. However, we found no association of SSB consumption frequency at 13 years with adiposity. Second, we have no information on total energy intake or amount of SSB intake, because we did not collect comprehensive detailed dietary information but only food frequency information. We have no information on the consumption of specific types of SSB, thus the reported SSB may include beverages sweetened with sugar or non-nutritive sweeteners. We assessed only canned SSB, which may have excluded those in bottles or cups and lead to the underreporting of SSB intake. Third, hydration status was not controlled for in the measurement of BFP. However, BFP was measured in the morning in healthy participants after an overnight fast, which likely reduces measurement error due to hydration status, illness, alcohol use or intense exercise. Fourth, only about $40 \%$ of the cohort were included, so selection bias is possible. However, those included and excluded were similar and we used IPW to recover the original sample. Fifth, variability in measures of adiposity was limited and few were obese $(\sim 4 \%)$, potentially reducing power. We only have $80 \%$ power to detect an effect size of 0.09 or greater in BMI z-score (i.e., BMI increase of $0.3 \mathrm{~kg} / \mathrm{m}^{2}$ or greater) for $1-3$ times or more per week as compared to less than weekly, thus we cannot exclude a small effect of SSB on adiposity [5,30], or an effect of SSB for overweight and obese children.

SSB consumption is seen as an important target of intervention to combat obesity in Western countries. However, added sugars and SSB intake have declined while obesity has risen steeply over the same time period in Australia, which to some extent challenges the assumption that reduced consumption of SSB can, in itself, help reverse the upward trend of obesity [38]. SSB consumption, as a marker of an unhealthy lifestyle characterized by more energy intake, less exercise, and a poor dietary pattern [39], alone may not dramatically contribute to obesity over time, but rather a constellation of structural, environmental and personal factors that impact long-term weight gain. Still, the overconsumption of SSB remains an important risk factor for the development of obesity in Western children with high SSB intake and strategies targeting SSB as a source of excess energy appear to be prudent [39]. Better understanding of the role of SSB intake in obesity in different populations would ensure policy initiatives are invested in effective targets of intervention. 


\section{Conclusions}

In Hong Kong Chinese children with low SSB consumption and less clear socioeconomic patterning of SSB consumption and obesity, SSB consumption frequency was unrelated to adiposity. Although we cannot rule out the possibility of small effects of SSB, especially for overweight and obese children, our study highlights the role of evidence from different social contexts in examining empirically driven hypotheses from long-term economically developed populations.

Supplementary Materials: The following are available online at http://www.mdpi.com/2072-6643/12/4/1015/s1, Figure S1: Participant flow chart in the "Children of 1997" Birth Cohort, Table S1: Characteristics of children with $(n=3628)$ and without $(n=4670)$ information on SSB consumption at 11 years in "Children of 1997" birth cohort, Table S2: Associations of SSB consumption at 11 years with BMI z-score, BMI centile and overweight (including obesity) from 12 to 18 years (complete case analysis), Table S3: Associations of SSB consumption at 11 years with WC, WHR and BFP at 16-19 years (complete case analysis), Table S4: Misclassification of SSB consumption at 11 years (complete case analysis), Table S5: Associations of composite SSB consumption at 11 and 13 years with BMI z-score and overweight from 12\#x2013;18 years.

Author Contributions: C.M.S. and G.M.L. designed and supervised the "Children of 1997" birth cohort. C.M.S. conceptualized the study, directed the analytic strategy and supervised the study from conception to completion, with assistance from S.L.A.Y., M.K.K. and L.L.H. T.Z. analyzed the data and drafted the manuscript. C.M.S., G.M.L., S.L.A.Y., M.K.K. and L.L.H. critically revised the manuscript. C.M.S. had primary responsibility for final content. All the authors contributed to the interpretation of the data, review of the paper and approval of the final version. All authors have read and agreed to the published version of the manuscript.

Funding: The "Children of 1997" birth cohort that was initially supported by the Health Care and Promotion Fund, Health and Welfare Bureau, Government of the Hong Kong SAR (HCPF grant 216106) and reestablished in 2005 with support from the Health and Health Services Research Fund (HHSRF grant 03040771) and The Research Fund for the Control of Infectious Diseases (RFCID 04050172) Government of the Hong Kong SAR. The Biobank Clinical Follow-up was partly funded by the WYNG Foundation. The funding body had no role in the design of the study and collection, analysis, and interpretation of data and in writing the manuscript.

Acknowledgments: The authors thank colleagues at the Student Health Service and Family Health Service of the Department of Health for their assistance and collaboration. They also thank the late Connie O for coordinating the project and all the fieldwork for the initial study in 1997-1998.

Conflicts of Interest: The authors declare that they have no conflict of interest.

\section{Abbreviations}

$\begin{array}{ll}\text { ANOVA } & \text { one-way analysis of variance } \\ \text { BFP } & \text { body fat percentage } \\ \text { BMI } & \text { body mass index } \\ \text { CI } & \text { confidence intervals } \\ \text { GEE } & \text { generalized estimating equations } \\ \text { IPW } & \text { inverse probability weighting; } \\ \text { IVW } & \text { inverse variance weighting } \\ \text { MI } & \text { multiple imputation } \\ \text { OR } & \text { odds ratios } \\ \text { RCT } & \text { randomized controlled trials } \\ \text { SEP } & \text { socioeconomic position } \\ \text { SSB } & \text { sugar-sweetened beverage } \\ \text { WC } & \text { waist circumference } \\ \text { WHO } & \text { World Health Organization } \\ \text { WHR } & \text { waist-to-hip ratio. }\end{array}$

\section{References}

1. NCD Risk Factor Collaboration (NCD-RisC). Worldwide trends in body-mass index, underweight, overweight, and obesity from 1975 to 2016: A pooled analysis of 2416 population-based measurement studies in 128.9 million children, adolescents, and adults. Lancet 2017, 390, 2627-2642. [CrossRef]

2. DiMeglio, D.P.; Mattes, R.D. Liquid versus solid carbohydrate: Effects on food intake and body weight. Int. J. Obes. Relat. Metab. Disord. 2000, 24, 794-800. [CrossRef] [PubMed] 
3. World Health Organization. Guideline: Sugars Intake for Adults and Children; World Health Organization: Geneva, Switzerland, 2015.

4. Vos, M.B.; Kaar, J.L.; Welsh, J.A.; Van Horn, L.V.; Feig, D.I.; Anderson, C.A.M.; Patel, M.J.; Munos, J.C.; Krebs, N.F.; Xanthakos, S.A.; et al. Added Sugars and Cardiovascular Disease Risk in Children: A Scientific Statement from the American Heart Association. Circulation 2017, 135, e1017-e1034. [CrossRef] [PubMed]

5. Malik, V.S.; Pan, A.; Willett, W.C.; Hu, F.B. Sugar-sweetened beverages and weight gain in children and adults: A systematic review and meta-analysis. Am. J. Clin. Nutr. 2013, 98, 1084-1102. [CrossRef]

6. Te Morenga, L.; Mallard, S.; Mann, J. Dietary sugars and body weight: Systematic review and meta-analyses of randomised controlled trials and cohort studies. BMJ 2012, 346, e7492. [CrossRef]

7. Ioannidis, J.P.A. The Challenge of Reforming Nutritional Epidemiologic Research. JAMA 2018, 320, 969-970. [CrossRef]

8. Peace, K.E.; Yin, J.; Rochani, H.; Pandeya, S.; Young, S. A Serious Flaw in Nutrition Epidemiology: A Meta-Analysis Study. Int. J. Biostat. 2018, 14. [CrossRef]

9. de Ruyter, J.C.; Olthof, M.R.; Seidell, J.C.; Katan, M.B. A trial of sugar-free or sugar-sweetened beverages and body weight in children. N. Engl. J. Med. 2012, 367, 1397-1406. [CrossRef]

10. Ebbeling, C.B.; Feldman, H.A.; Chomitz, V.R.; Antonelli, T.A.; Gortmaker, S.L.; Osganian, S.K.; Ludwig, D. A randomized trial of sugar-sweetened beverages and adolescent body weight. N. Engl. J. Med. 2012, 367, 1407-1416. [CrossRef]

11. Kaiser, K.A.; Shikany, J.M.; Keating, K.D.; Allison, D.B. Will reducing sugar-sweetened beverage consumption reduce obesity? Evidence supporting conjecture is strong, but evidence when testing effect is weak. Obes. Rev. 2013, 14, 620-633. [CrossRef]

12. Mattes, R.D.; Shikany, J.M.; Kaiser, K.A.; Allison, D.B. Nutritively sweetened beverage consumption and body weight: A systematic review and meta-analysis of randomized experiments. Obes. Rev. 2011, 12, 346-365. [CrossRef] [PubMed]

13. Auerbach, B.J.; Wolf, F.M.; Hikida, A.; Vallila-Buchman, P.; Littman, A.; Thompson, D.; Louden, D.; Taber, D.R.; Krieger, J. Fruit Juice and Change in BMI: A Meta-analysis. Pediatrics 2017, 139, e20162454. [CrossRef] [PubMed]

14. Au Yeung, S.L.; Schooling, C.M. More ways to distinguish real from artefactual associations in observational studies. Int. J. Epidemiol. 2014, 43, 1665-1666. [CrossRef] [PubMed]

15. Schooling, C.M.; Yau, C.; Cowling, B.J.; Lam, T.H.; Leung, G.M. Socio-economic disparities of childhood Body Mass Index in a newly developed population: Evidence from Hong Kong's 'Children of 1997' birth cohort. Arch. Dis. Child. 2010, 95, 437-443. [CrossRef]

16. Shrewsbury, V.; Wardle, J. Socioeconomic status and adiposity in childhood: A systematic review of cross-sectional studies 1990-2005. Obesity (Silver Spring) 2008, 16, 275-284. [CrossRef]

17. Gui, Z.H.; Zhu, Y.N.; Cai, L.; Sun, F.H.; Ma, Y.H.; Jing, J.; Chen, Y.J. Sugar-Sweetened Beverage Consumption and Risks of Obesity and Hypertension in Chinese Children and Adolescents: A National Cross-Sectional Analysis. Nutrients 2017, 9, 1302. [CrossRef] [PubMed]

18. van Ansem, W.J.; van Lenthe, F.J.; Schrijvers, C.T.; Rodenburg, G.; van de Mheen, D. Socio-economic inequalities in children's snack consumption and sugar-sweetened beverage consumption: The contribution of home environmental factors. Br. J. Nutr. 2014, 112, 467-476. [CrossRef]

19. Schooling, C.M.; Hui, L.L.; Ho, L.M.; Lam, T.-H.; Leung, G.M. Cohort Profile: 'Children of 1997': A Hong Kong Chinese birth cohort. Int. J. Epidemiol. 2012, 41, 611-620. [CrossRef]

20. Sun, Y. Diet and Cardiovascular Disease Risk in Chinese. Ph.D. Thesis, The University of Hong Kong, Hong Kong, China, 2015.

21. Lin, S.L.; Tarrant, M.; Hui, L.L.; Kwok, M.K.; Lam, T.H.; Leung, G.M.; Schooling, C.M. The role of dairy products and milk in adolescent obesity: Evidence from Hong Kong's "Children of 1997" birth cohort. PLOS ONE 2012, 7, e52575. [CrossRef]

22. de Onis, M.; Onyango, A.W.; Borghi, E.; Siyam, A.; Nishida, C.; Siekmann, J. Development of a WHO growth reference for school-aged children and adolescents. Bull. World Health Organ. 2007, 85, 660-667. [CrossRef] [PubMed]

23. Cole, T.J.; Bellizzi, M.C.; Flegal, K.M.; Dietz, W.H. Establishing a standard definition for child overweight and obesity worldwide: International survey. BMJ 2000, 320, 1240-1243. [CrossRef] [PubMed] 
24. Cole, T.J.; Lobstein, T. Extended international (IOTF) body mass index cut-offs for thinness, overweight and obesity. Pediatr. Obes. 2012, 7, 284-294. [CrossRef] [PubMed]

25. Cohen, J. Statistical Power Analysis for the Behavioral Sciences, 2nd ed.; Lawrence Erlbaum Associates: Hillsdale, NJ, USA, 1988.

26. Schafer, J.L. Multiple imputation: A primer. Stat. Methods Med. Res. 1999, 8, 3-15. [CrossRef] [PubMed]

27. Seaman, S.R.; White, I.R.; Copas, A.J.; Li, L. Combining multiple imputation and inverse-probability weighting. Biometrics 2012, 68, 129-137. [CrossRef] [PubMed]

28. Shang, X.W.; Liu, A.L.; Zhang, Q.; Hu, X.Q.; Du, S.M.; Ma, J.; Xu, G.F.; Li, Y.; Guo, H.W.; Du, L.; et al. Report on childhood obesity in China (9): Sugar-sweetened beverages consumption and obesity. Biomed. Environ. Sci. 2012, 25, 125-132. [CrossRef]

29. Marshall, T.A.; Curtis, A.M.; Cavanaugh, J.E.; Warren, J.J.; Levy, S.M. Child and Adolescent Sugar-Sweetened Beverage Intakes Are Longitudinally Associated with Higher Body Mass Index z Scores in a Birth Cohort Followed 17 Years. J. Acad. Nutr. Diet. 2019, 119, 425-434. [CrossRef]

30. Vartanian, L.R.; Schwartz, M.B.; Brownell, K.D. Effects of soft drink consumption on nutrition and health: A systematic review and meta-analysis. Am. J. Public Health 2007, 97, 667-675. [CrossRef]

31. Malik, V.S.; Willett, W.C.; Hu, F.B. Sugar-sweetened beverages and BMI in children and adolescents: Reanalyses of a meta-analysis. Am. J. Clin. Nutr. 2009, 89, 439-440. [CrossRef]

32. Slining, M.M.; Popkin, B.M. Trends in intakes and sources of solid fats and added sugars among U.S. children and adolescents: 1994-2010. Pediatr. Obes. 2013, 8, 307-324. [CrossRef]

33. Perrar, I.; Schadow, A.M.; Schmitting, S.; Buyken, A.E.; Alexy, U. Time and age trends in free sugar intake from food groups among children and adolescents between 1985 and 2016. Nutrients 2019, 12, 20. [CrossRef]

34. Hu, S.; Wang, L.; Yang, D.; Li, L.; Togo, J.; Wu, Y.; Liu, Q.; Li, B.; Li, M.; Wang, G.; et al. Dietary Fat, but Not Protein or Carbohydrate, Regulates Energy Intake and Causes Adiposity in Mice. Cell Metab. 2018, 28, 415-431.e4. [CrossRef] [PubMed]

35. Jing, Y.; Han, T.S.; Alkhalaf, M.M.; Lean, M.E.J. Attenuation of the association between sugar-sweetened beverages and diabetes risk by adiposity adjustment: A secondary analysis of national health survey data. Eur. J. Nutr. 2018, 58, 1703-1710. [CrossRef] [PubMed]

36. Woo, J.; Leung, S.S.F.; Ho, S.C.; Lam, T.H.; Janus, E.D. A food frequency questionnaire for use in the Chinese population in Hong Kong: Description and examination of validity. Nutr. Res. 1997, 17, 1633-1641. [CrossRef]

37. Foster, E.; Bradley, J. Methodological considerations and future insights for 24-hour dietary recall assessment in children. Nutr. Res. 2018, 51, 1-11. [CrossRef] [PubMed]

38. Brand-Miller, J.C.; Barclay, A.W. Declining consumption of added sugars and sugar-sweetened beverages in Australia: A challenge for obesity prevention. Am. J. Clin. Nutr. 2017, 105, 854-863. [CrossRef] [PubMed]

39. Khan, T.A.; Sievenpiper, J.L. Controversies about sugars: Results from systematic reviews and meta-analyses on obesity, cardiometabolic disease and diabetes. Eur. J. Nutr. 2016, 55 (Suppl. 2), 25-43. [CrossRef] [PubMed]

(C) 2020 by the authors. Licensee MDPI, Basel, Switzerland. This article is an open access article distributed under the terms and conditions of the Creative Commons Attribution (CC BY) license (http://creativecommons.org/licenses/by/4.0/). 